\title{
Treatment of prostate cancer: therapeutic potential of targeted immunotherapy with APC80I5
}

\author{
Robert A Bok \\ Department of Radiology, University \\ of California at San Francisco, San \\ Francisco, CA, USA
}

\begin{abstract}
The body's immune system has some capacity to recognize and attack cancerous growths, including prostate cancer. However, various intrinsic characteristics of tumor cells usually limit that capacity. Therapeutically administered immunologic stimuli, such as APC8015, an individualized, ex vivo stimulation of a patient's own antigen presenting cells (APC), are capable of boosting the anti-tumor response. Late phase clinical trials of APC8015 (now also called Sipuleucel-T) show evidence of slowing disease progression and increasing survival in advanced prostate cancer. Such immunotherapeutic approaches hold real promise to provide additional useful and welcome weapons against this common malignancy.
\end{abstract}

Keywords: prostate cancer, antigen presenting cells, immunologics

\section{Therapeutic challenge and existing clinical practice}

Prostate cancer (PC) was diagnosed in about 234,000 men and responsible for over 27,000 deaths within the United in 2006 (Jemal et al 2006), while 219,000 cases and another 27,000 deaths are estimated for 2007 (Jemal et al 2007). Although definitive therapy (surgery and/or radiation) can cure the majority of men with localized disease, some $20 \%-30 \%$ of treated men will relapse and a substantial fraction of these will succumb to this malignancy. Androgen-ablation therapy with luteinizinghormone-releasing hormone agonists and anti-androgens is the mainstay of treatment for such patients, but is not curative and eventually fails. After first-line hormonal agents fail, additional hormone-modulating therapies are usually explored. When patients become truly hormone-refractory, taxane chemotherapy is the most accepted treatment. Docetaxol (Taxotere ${ }^{\circledR}$ )-based chemotherapy has proven survival benefit, but can be too toxic for some patients and also is non-curative treatment (Tannock et al 2004). Ultimately, unfortunately, these second and third-line hormonal therapies and chemotherapies fail and subsequent options for relapsed patients are limited, focusing primarily on palliative measures (Ross and Kantoff 2007). Consequently, there is a great need for newer, more effective yet relatively non-toxic systemic therapies for PC.

Immunologic or vaccine-type treatments have the potential, theoretically, to meet this need. The challenge with this type of therapeutic approach is to elicit and sustain a sufficiently robust immunologic response to one's own tumor cells, while leaving most other normal cells in the body unaffected. (Normal prostate cells are excluded here, because preservation of normal prostatic epithelium for PC patients is not necessarily a desirable thing). The main goal of immunologic treatments, therefore, is to provide therapeutic benefit from the vaccination without creating a deleterious autoimmune response. This is inherently problematic given the "self-like" character of cancer cells. While they are clearly dysregulated physiologically and different histologically from the normal tissues cell from which they arise (epithelial cells in the case of prostatic 
carcinoma), tumor cells have an antigenic makeup that is difficult to distinguish from that of their normal counterparts. PC cells in particular are generally considered to be poorly immunogenic (Saika et al 2004). Means have thus been sought to bolster the immune system's ability to recognize features of PC cells which are unique and sufficiently different from normal cells, so as to overcome tumor tolerance.

Ways to stimulate the body's own immune system to fight cancerous growths have long been sought and various methods have been explored and adopted over the past century. Early efforts employed the instillation of inflammatory/ immunogenic microbes into the tumor environment (Coley 1893). Coley and others applied this strategy successfully against a number sarcomas and carcinomas over a century ago. Early stage, non-invasive bladder cancers are frequently treated today with such microbe-based therapy. Infusion of Bacille Calmette-Guerin (BCG) into the bladder elicits a nonspecific immune response which can control early bladder cancers (Karnes et al 2006). More recent efforts have focused on use of specific cytokines to stimulate immune recognition of cancer cells. Perhaps the best publicized attempts relate to investigations (reviewed in Rosenberg (2001)) at the NCI in the 1980's. High-dose interleukin-2 (IL-2) and isolated tumor infiltrating lymphocytes (TIL) cells and, later, low-dose IL-2 and interferon showed some promise of controlling certain types of cancer, including renal cell carcinoma. The use of immunotherapies in urologic malignancies, therefore, has solid historical precedence, having been employed against kidney and bladder cancer for some time.

\section{Developmental history}

The newer vaccine therapies, such as APC8015, have been built upon the previous efforts mentioned above coupled with improved knowledge of the molecular regulators of immunity. Indeed, like most medical advances, development of this vaccine therapy is the culmination of stepwise progress in several related areas of research. One area has been advances in understanding of antigen presenting cells (APC), their maturation and roles in immune surveillance and response to antigens. Another has been improved knowledge of cytokines and receptors regulating the immune response. Another has been technical improvements in identifying and isolating APC for therapeutic manipulation.

\section{Antigen presenting cell}

APC are key players in the immunologic response to target antigens, expressing costimulatory molecules as well as immune-activating cytokines (IL-12, GM-CSF, TNF- $\alpha$, etc.) to elicit both primary and secondary immune responses (Saika et al 2004). APC can include activated B lymphocytes, monocytes, and macrophages, but dendritic cells (DC) are considered the most potent antigen presenters, capable of initiating anti-tumor responses from both naïve and memory T-cells (Karnes et al 2006). DC were first described in 1973 by Steinmann and Cohn. These investigators delineated cells in the spleen and lymph nodes of mice, which turned out to have extensive cytoplasmic process under light and electron microscopy. These cells were subsequently shown to internalize, process and then display foreign antigens to $\mathrm{B}$ and $\mathrm{T}$ lymphocytes and thus to be critical for priming cytotoxic $\mathrm{T}$ lymphocyte(CTL)-mediated immune response (Mayordoma et al 1997). By the mid-1990's APC were being experimentally manipulated to treat $\mathrm{PC}$ in preclinical studies (Fong et al 1995).

In conjunction with the above studies, improvements in the identification of APC and various categories of APC have also occurred (Wilkinson et al 2006). Advances have also been made in the techniques used to isolate, culture and expand APC/DC from human patients, such as density gradient centrifugation (Hsu et al 1996) and various leukapheresis methods (Mayordoma et al 1997).

\section{GM-CSF}

A number of protein factors have been identified that regulate APC in both a positive and negative manner. Granulocyte macrophage colony stimulating factor (GM-CSF) was originally identified and cloned because of its ability to stimulate white blood cell production in leukopenic patients. It was shown subsequently to be one of the most potent stimulators of APC, enhancing their differentiation, survival, and possibly even antigen processing ability (Rini et al 2006 and references therein). GM-CSF seems to have the most profound effects on DC functions (Sallusto et al 1994), compared to other factors, such as interferon- $\alpha$, IL-12, and CD40 ligand (Klein et al 2000).

In preclinical studies, which utilized the Dunning rat model for PC, vaccination with GM-CSF gene-modified tumor cells resulted in longer disease-free survival compared to untreated animals (Vieweg et al 1994). A preclinical study by Hurwitz et al (2000) in a transgenic mouse model for PC, using GM-CSF-augmented vaccination and another immunomodulator, yielded similar results. Stimulation of the immune system by exogenous GM-CSF has shown promise in clinical studies of several malignancies. Spitler et al (2000) reported its efficacy as a single adjuvant agent against melanoma. Other clinical investigations with recombinant GM-CSF as 
a sole agent also showed evidence of benefit in certain PC patients (Small et al 1999).

\section{Pharmacology/immunology}

Srivastava (2006) has pointed out the important distinction between prophylactic vaccination versus therapeutic vaccination. The former, of course, has been used extensively and extraordinarly successfully to combat infectious diseases around the world. The latter category, however, is more pertinent to the arena of oncological therapy and, unfortunately, more difficult to achieve. This difficulty arises from two-factors: first is the innate ability of tumor cells to escape immunologic detection; the second is the need to elicit a sufficiently robust cytotoxic response to a usually already well-established, substantial volume of disease (in contrast to the situation with prophylactic vaccination against a newly invading infectious organism). Mechanisms by which tumor cells escape from the body's immune responses are several and include: (1) down regulation of the immune response by tumor cells themselves or by factors that they release; (2) altered expression of the major histocompatibility complex proteins by the tumor cells, impairing immune recognition; (3) altered expression of immunoregulatory factors and/or adhesion molecules by tumor or DC (eg, increased vascular endothelial growth factor (VEGF) expression); and (4) subversion of the immune response elements to actually facilitate tumor cell growth (Pawlec et al 2000; Karnes et al 2006).

As described earlier, central players in both the humoral and the cellular arms of the immune response are the APC, particularly the so-called DC. In the 1990's, a number of different research groups reported success with DC manipulation to enhance antitumor immunologic response. DC are considered the most potent of the APC, capable of initiating T-cell-dependent immune responses from naïve T cells (Steinman et al 1991; Hart, 1997). Several immuneactivating cytokines are able to modulate DC function within the local tissue environment, including interferon- $\gamma$, TNF- $\alpha$, IL-12 and GM-CSF (Mayordorma et al 2006). GM-CSF is a critical regulatory factor for several immune pathways (Schwaab et al 2006). It is a particularly potent stimulator of DC (Sallusto et al 1994). By the mid-1990's, several investigators reported efficient tumor suppression and survival benefit in mouse models, using GM-CSF-transduced tumor vaccines (Dranoff et al1993; Abe et al 1995). More recently, it was demonstrated that genetically modified DC expressing GM-CSF elicit a specific cytotoxic T-cell response and therapeutic immunity in murine tumor models (CurielLewandrowski, 1999; Nakamura et al 2006).
For APC8015, the tissue-specific protein chosen by Dendreon as the target antigen for immunization was prostatic acid phosphatase (PAP). Once utilized as a tumor marker for PC (before the advent of PSA), PAP is expressed in about $95 \%$ of PC tumors, has fairly specific expression for prostatic tissue, and so was considered a good candidate for a prostate cancer-targeting antigen. To enhance its antigenicity and DCstimulating properties, PAP was linked to GM-CSF, using recombinant DNA techniques, producing a fusion protein termed PA2024. PA2024 thus consists of human PAP fused through its carboxy terminus to the amino terminus of human GM-CSF via a Gly-Ser linker. It is expressed in a Baculovirus system and purified to $>90 \%$ purity by a procedure that has been described (Burch et al 2000).

APC8015 (Sipuleucel-T) therefore consists essentially of an individual patient's APC primed by exposure to PA2024. The final vaccine product is produced by isolating an individual patient's CD54-positive white cells via a leukapheresis procedure, exposing the isolated cells ex vivo to PA2024, incorporating the stimulated cells into Dendreon's proprietary antigen delivery cassette, and infusing the vaccine back into the patient intravenously. The vaccine is given on several different occasions, usually three times over a 1 -month period. APC8015 is thus comprised of autologous APC, but is a mixed cell suspension containing also monocytes, macrophages, B and T cells, loaded with PA2024 (Burch et al 2004).

Pharmacologically, the development of APC8015 entails some unique challenges. It is comprised of a recombinant antigenic protein which must be incubated with an individual patient's isolated APC ex vivo. Thus, its preparation is a multi-step process that requires extraction of blood cells from the patient, transporting them to the vaccine manufacturer, incubating them with recombinant antigen, ensuring sterility of the processed cells, and returning them to the patient/doctor for infusion (Figure 1). The FDA has not approved a therapeutic cancer vaccine to date. Manufacturing a product that is customized to each individual patient, with the consistency and purity demanded by the FDA, is a significant hurdle to clear. In September 2003, however, the FDA designated APC8015 to an accelerated development program and subsequently granted the vaccine fast-track status in November 2005.

\section{Animal/early human studies}

A variety of preclinical studies with infused APC/DC, employing GM-CSF as a stimulatory factor and a tumor antigen for targeting, showed good efficacy against various 


\section{Sipuleucel-T (Provenge ${ }^{\circledR}$ ) Manufacturing Process}
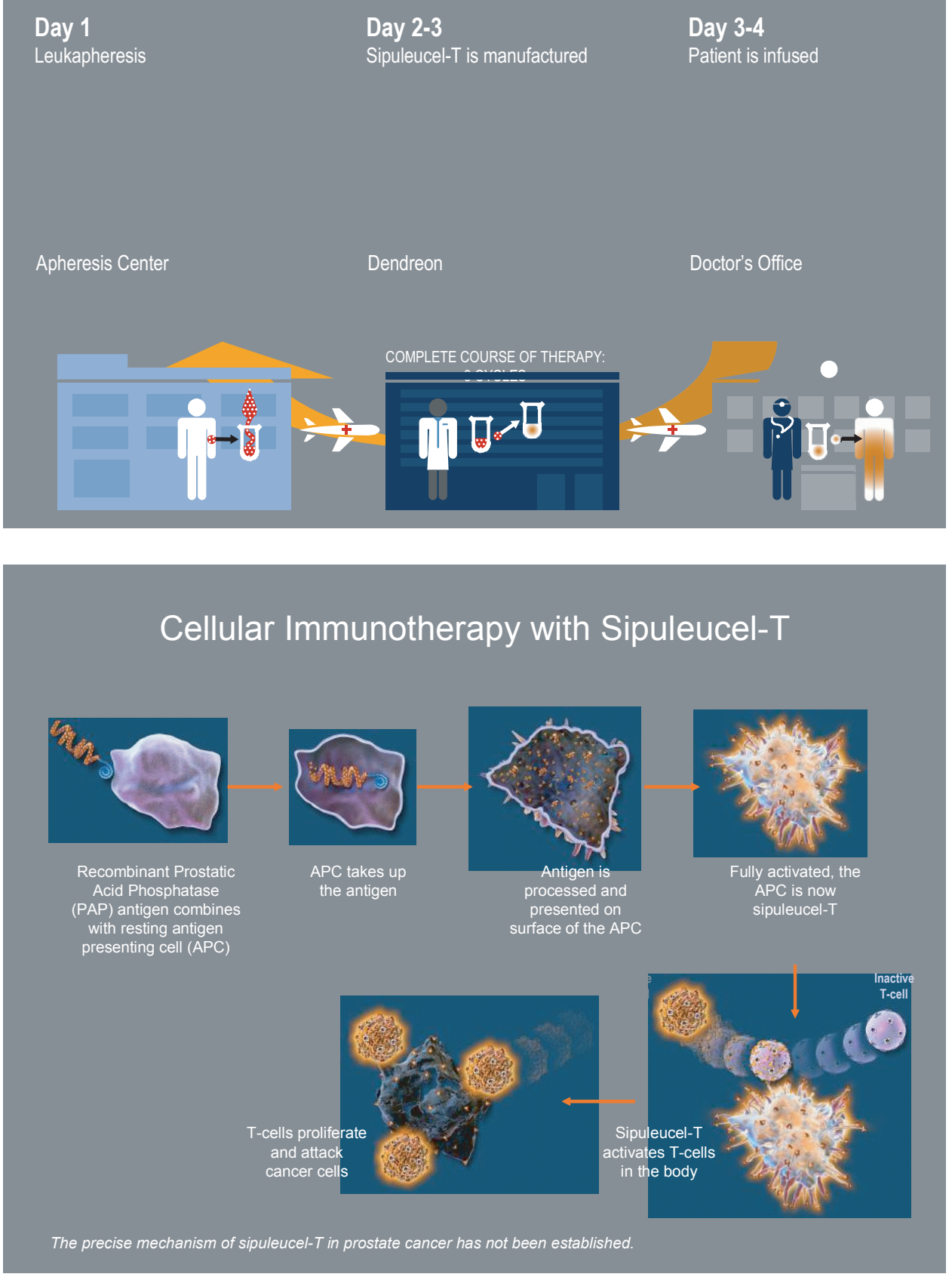

Figure I From Dendreon presentation to FDA.

cancers in animal models. Improvement in both humoral (Flamand et al 1999) and cellular immune responses (Celluzzi et al 1996; Curiel-Lewandrowski et al 1999; Nakamura et al 2002) occur with this vaccination strategy, though the later appears more critical. There are numerous published animal studies showing that specific CTL stimulation is facilitated by GM-CSF. Human studies have demonstrated this subsequently, also (Schwaab et al 2005). Mayordomo et al (1995) used ex vivo priming of DC with tumor-associated antigen in three different murine tumor models and showed 
excellent protection against tumor challenge in all, as well as an ability to eradicate pre-established tumors in two of the models.

\section{Review of clinical studies to date}

The first Phase I clinical trial of Dendreons's APC8015 was conducted at the Mayo Clinic between 1997-1998. This study enrolled 13 patients with hormone-refractory, metastatic PC, who were treated with APC8015 and then had several followup injections of PA2024 alone. Treatment with APC8015 was tolerated well, with only mild (grade 1-2), transient episodes of fever, chills, myalgia, pain and fatigue reported. Three of these patients had a drop in their PSA level by $50 \%$ or more. All patients exhibited evidence of immunological response to treatment (Burch et al 2000). The next clinical trial with Dendreon's APC8015 was a combined Phase I and II clinical study carried out in a group of 31 patients at the University of California, San Francisco, in the late 1990s. Again, all patients developed evidence of an immunologic response to the fusion protein PA2024 (Small et al 2000). However, only $20 \%$ of patients exhibited evidence of a tumor response, as measured by a drop in PSA of $25 \%$ or more from baseline.

A second Phase II study was conducted by Burch et al (2004). This group at the Mayo Clinic investigated a slightly smaller cohort of 21 patients with hormone-refractory, metastatic PC and found a transient PSA response in only $10 \%$ of participants. However, one of these was an individual who had a complete response radiographically, which was incredibly durable, lasting more than 4 years. The treatment was again generally very well tolerated in both of these Phase II studies, with the most common adverse effects being mild, grade $1-2$ rigors and fatigue.

The results from the early phase trials were sufficiently promising to warrant further investigation in a randomized, controlled study. The first Phase III study, designated D9901, was initiated in 2000 and enrolled 127 patients with asymptomatic, metastatic, hormone-refractory PC from 19 centers. The trial was completed in 2005. This placebo-controlled study showed no significant delay in time to disease progression (TTP) for the group as a whole $(\mathrm{p}$-value $=0.061)$, but did demonstrate a significance delay in TTP in a subset of patients who's tumors had a Gleason score of $\leq 7$ (a standard pathological grading scheme for PC ranging from 2 to 10). The secondary endpoint of the study, overall survival, also showed a significant difference. In the final three-year follow up of D9901, a median survival benefit of 21 percent or 4.5 months (25.9 months for treated patients versus 21.4 months for placebo group; $p$-value $=0.01$ ) and a three-fold improvement in survival fraction at 36 months $(34 \%$ of treated patients alive versus $11 \%$ of placebo group; $\mathrm{p}$-value $=0.01$; hazard ratio $=1.7)$ were seen $($ Small et al 2006).

Meanwhile, a companion Phase III study called D9902 had been started, which was a double-blinded, placebocontrolled study in asymptomatic, metastatic hormonerefractory PC patients. Ninty-eight patients were recruited by December 2002 when the study was halted due to the findings in D9901 related to the Gleason score subgroups. The first part of the study was then designated D9902A (the first 98 patients enrolled without regard to Gleason score). The protocol was subsequently amended to focus on patients with Gleason score of $\leq 7$ and continued as D9902B, again enrolling asymptomatic, metastatic hormone-refractory PC patients. This study began in July 2003, and is still underway, having an accrual target of 500 patients. The primary endpoint of D9902A, which again was TTP, did not show statistical significance unfortunately $(\mathrm{p}$-value $=0.033)$. An integrated analysis of D9901 and D9902A, however, did show a survival advantage in the overall intent to treat population (Srivastava 2006). Log rank analysis of the whole patient population showed a hazard ratio of $1.5(\mathrm{p}$-value $=0.011)$ favoring treatment with APC8015. Proportional hazards regression (Cox) analysis also revealed a favorable hazard ratio of 1.8 , with a p-value of 0.0006 (Hamawy 2006). In all human studies to date, the APC8015 treatment was well tolerated, with no dose-limiting toxicities observed (Small et al 2006). The main toxicities noted have continued to be mild, consisting of infusion-related, low-grade fever and chills lasting for one to two days.

In January 2007, Dendreon Corporation announced that the FDA accepted their application for a biologics license and assigned it priority review status. Priority review is granted to products that have potential to provide a significant improvement in the safety or effectiveness of the treatment, diagnosis or prevention of a serious or life-threatening disease. In March 2007, an advisory committee to the FDA reviewed clinical safety and efficacy data and voted in favor of both the safety and efficacy of APC8015. However, in May 2007, the FDA decided that the available data was insufficient for approval at that time (which has generated some controversy among PC advocacy groups). The agency has requested further clinical data to support the efficacy claims in the Biologics License Application, as well as further information on the chemistry, manufacture and controls for APC8015 production. A final decision by the FDA is thus still pending. Interim survival results from the ongoing Phase III study (D9902B) are expected by Dendreon sometime in 2008. 


\section{Conclusions and future directions}

The clinical trials with APC8015 to date indicate a moderate degree of immunological response that provides some therapeutic benefit. The level of response, however, is not as robust in many patients, as might be hoped for. Additional data on the true magnitude of the response will become clearer as the latest Phase III trials mature. Nevertheless, it appears that additional manipulations of the immune system may be required to elicit the greatest potential benefit from this immunological therapeutic approach. Subsequent clinical trials will probably focus on the combination of APC8015 with other biologicals, especially additional immunoregulatory factors, to enhance the anti-tumor effect of the immune response. This has already begun with the investigation of Bevacizumab, a monoclonal antibody against VEGF, in combination with APC8015. Although originally developed because of its anti-angiogenesis properties, Bevacizumab (anti-VEGF) can also positively modulate the immune system, because VEGF has a negative (suppressive) regulatory effect on DC (Oyama et al 1998). This coordinated administration of anti-VEGF to patients receiving APC8015 vaccine has potential to further enhance the immune stimulation of the PA2024 primed dendritic cells. Indeed, a Phase II study of APC8015 in combination with bevacizumab among patients with hormone-dependent PC has been undertaken by the National Cancer Institute. Other combinations are undoubtedly under consideration and may include use of such recombinant stimulatory cytokines as IL-12 (Saika et al 2004), IL-2, Interferon, or anti-CTLA4 (CTLA4 is an endogenous dampening factor for APC and antibodies have been developed which inhibit its effects; Quezada et al 2006). Of course, other modulations of APC 8015 therapy might also be investigated, including increased number of infusions, coordination with standard hormonal therapies, or combination with standard radiation or even chemotherapies. For instance, androgen ablation therapy has marked effects on the degree and the nature of $\mathrm{T}$ cell responses within prostate tissue and could possibly augment immunotherapeutics for PC (Mercader et al 2006).

Discernable progress has been made in the development of immunologic therapies for PC, but additional work lies ahead before the full potential of this therapeutic approach will be realized.

\section{References}

Burch PA, Breen JK, Buckner JC, et al. 2000. Priming tissue-specific cellular immunity in a phase I trial of autologous dendritic cells for prostate cancer. Clin Cancer Res, 6:2175-82.
Burch PA, Croghan GA, Gastineau DA, et al. 2004. Immunotherapy (APC8015, Provenge ${ }^{\circledR}$ ) targeting prostatic acid phosphatase can induce durable remission of metastatic androgen-independent prostate cancer: a phase 2 trial. The Prostate, 60:107-204.

Coley WB. 1893. The treatment of malignant tumors by repeated inoculations of erysipelas: With a report of ten original cases. Am J Med Sci, 105:487.

Curiel-Lewandrowski C, Mahnke K, Labeur M, et al. 1999. Transfection of immature murine bone marrow-derived dendritic cells with the granulocyte-macrophage colony-stimulating factor gene potently enhances their in vivo antigen-presenting capacity. $J$ Immunol, 163:174-83.

Fong L, Brockstedt D, Benike C, et al. 2001. Dendritic cell-based xenoantigen vaccination for prostate cancer immunotherapy. J Immunol, 167:7150-6.

Hamawy KJ, Libertino J, Frohlich M. 2006. Improved survival with Sipuleucel-T (Apc8015) in men with advanced prostate cancer. American Urological Assoc 75th Annual Meeting.

Hart DN. 1997. Dendritic cells: unique leukocyte populations which control the primary immune response. Blood, 90:3245-87.

Hurwitz AA, Foster BA, Kwon ED, et al. 2000. Combination immunotherapy of primary prostate cancer in a transgenic mouse model using CTLA-4 blockade. Cancer Res, 60:2444-8.

Hsu FJ, Benike C, Fagnoni F, et al. 1996. Vaccination of patients with B-cell lymphoma using autologous antigen-pulsed dendritic cells. Nat Med, 2:52-8.

Jemal A, Siegel R, Ward E, et al. 2006. Cancer Statistics. CA Cancer J Clin, 56:106-30.

Jemal A, Siegel R, Ward E, et al. 2007. Cancer statistics. CA Cancer J Clin, 57:43-66.

Klein C, Bueler H, Mulligan RC. 2000. Comparative analysis of genetically modified dendritic cells and tumor cells as therapeutic cancer vaccines. J Exp Med, 191:1699-708.

Mercader M, Bodner BK, Moser MT, et al. 2001. T cell infiltration of the prostate induced by androgen withdrawal in patients with prostate cancer. Proc Natl Acad Sci USA, 98:14565-70.

Nakamura M, Iwahashi M, Nakamori M, et al. 2002. Dendritic cells genetically engineered to simultaneously express endogenous tumor antigen and granulocyte macrophage colony-stimulating factor elicit potent therapeutic antitumor immunity. Clin Cancer Res, 8:2742-9.

Oyama T, Ran S, Ishida T, et al. 1998. Vascular endothelial growth factor affects dendritic cell maturation through the inhibition of nuclear factor-kB activation in hemopoietic progenitor cells. J Immunol, 160:1224-32.

Quezada SA, Peggs KS, Curran MA, et al. 2006. CTLA4 blockade and GM-CSF combination immunotherapy alters the intratumor balance of effector and regulatory T cells. J Clin Invest, 116:1935-45.

Rini BI, Fong L, Weinberg V, et al. 2006. Clinical and immunological characteristics of patients with serologic progression of prostate cancer achieving long-term disease control with granulocyte-macrophage colony-stimulating factor. J Urology, 175:2087-91.

Rosenberg SA. 2001. Progress in the development of immunotherapy for the treatment of patients with cancer. $J$ Intern Med, 250:462-75.

Ross RW, Kantoff PW. 2007. Hormone-refractory prostate cancer: choosing the appropriate treatment option. Oncology (Williston Park), 21:185-93.

Saika T, Satoh T, Kusaka N, et al. 2004. Route of administration influences the antitumor effects of bone marrow-derived dendritic cells engineered to produce interleukin-12 in a metastatic mouse prostate cancer model. Cancer Gene Therapy, 11:317-24.

Sallusto F, Lanzavecchia A. 1994. Efficient presentation of soluble antigen by cultured human dendritic cells is maintained by granulocyte/macrophage colony-stimulating factor plus interleukin 4 and downregulated by tumor necrosis factor. $J$ Exp Med, 179:1109-18.

Small EJ, Fratesi P, Reese DM, et al. 2000. Immunotherapy of hormonerefractory prostate cancer with antigen-loaded dendritic cells. J Clin Oncol, 18:3894-903. 
Small EJ, Schellhammer PF, Higano CS, et al. 2006. Placebo-controlled phase III trial of immunologic therapy with Sipuleucel-T (APC8015) in patients with metastatic, asymptomatic hormone refractory prostate cancer. J Clin Oncol, 24:3089-94.

Spitler LE, Grossbard ML, Ernstoff MS, et al. 2000. Adjuvant therapy of stage III and IV malignant melanoma using granulocyte-macrophage colony-stimulating factor. J Clin Oncol, 18:1614-21.

Srivastava PK. 2006. Therapeutic cancer vaccines. Curr Opin Immunol, $18: 201-5$.

Steinmann RM, Cohn ZA. 1973. Identification of a novel cell type in peripheral lymphoid organs of mice. I. Morphology, quantitation, tissue distribution. J Exp Med, 137:1142-62.

Tannock IF, de Wit R, Berry WR, et al. 2004. Docetaxel plus prednisone or mitoxantrone plus prednisone for advanced prostate cancer. $N$ Engl $J$ Med, 351:1502-12.

Wilkinson R, Kassianos AJ, Swindle P, et al. 2006. Numerical and functional assessment of blood dendritic cells in prostate cancer patients. The Prostate, 66:180-92. 
